\title{
Boundary Conditions for a Fourth Order Hyperbolic Difference Scheme
}

\author{
By D. M. Sloan
}

\begin{abstract}
Oliger [6] has used a stable time-averaged boundary condition with a fourth order leap-frog scheme for the numerical solution of hyperbolic partial differential equations. Gary [3] generalized the time-averaged boundary condition by including a scalar parameter. This paper examines the stability and accuracy of the more general boundary condition. The limit of the stability interval is found for the parameter, and it is shown that the parameter should be given a value close to this limit in order to minimize the boundary errors. Numerical experiments are described which support the theoretical predictions.
\end{abstract}

1. Introduction. Finite difference schemes often require more boundary conditions than the differential equations which they approximate. The additional boundary conditions may be constructed using a mathematical or a physical approach and, in either case, care has to be taken to avoid the introduction of boundary approximations which give rise to instabilities. Here we consider boundary approximations to be used for the numerical solution of hyperbolic partial differential equations. Of particular interest is the stability and accuracy of a class of boundary approximations when this class is conjoined with an interior difference scheme which uses second order leap-frog differences in time and fourth order centered differences in space.

An initial and boundary value difference scheme will be constructed as an approximation to the simple hyperbolic problem defined by

$$
\begin{gathered}
u_{t}=c u_{x}, \quad c>0,0 \leqslant x \leqslant 1, t \geqslant 0, \\
u(1, t)=g(t), \quad t \geqslant 0, \\
u(x, 0)=q(x), \quad 0 \leqslant x \leqslant 1,
\end{gathered}
$$

with the compatibility condition $g(0)=q(1)$ satisfied. Suppose $u$ is approximated by a grid function $U_{\Delta}$, and let $U_{j}^{n}$ denote the value of $U_{\Delta}$ at the grid point $x=x_{j}=j \Delta x$, $t=t_{n}=n \Delta t$, where $\Delta x=1 / J$ and $J$ is a positive integer. The approximation to (1.1), with accuracy $O\left(\Delta t^{2}+\Delta x^{4}\right)$, may be written as

$$
U_{j}^{n+1}=U_{j}^{n-1}+\frac{\lambda}{6}\left(U_{j-2}^{n}-8 U_{j-1}^{n}+8 U_{j+1}^{n}-U_{j+2}^{n}\right), \quad j=2,3, \ldots, J-2,
$$

where $\lambda=c \Delta t / \Delta x$, and the boundary condition (1.2) becomes

$$
U_{J}^{n+1}=g\left(t_{n+1}\right) .
$$

Received July 30, 1982.

1980 Mathematics Subject Classification. Primary 65M10.

Key words and phrases. Hyperbolic equations, finite differences, stability of boundary approximations. 
Additional boundary conditions are required for the evaluation of $U_{J}^{n+1} \cdot U_{0}^{n+1}$ and $t^{\prime \prime}$. and Oliger [6] has shown that a stable scheme is obtained using the supplementary conditions

(1.0) $U_{J}^{n} i_{1}^{1}=U_{J}^{n-1}+\frac{\lambda}{6}\left[4 U_{J}^{n}+3\left(U_{J}^{n+1}+U_{J}^{n}{ }_{1}^{1}\right)-12 U_{J-2}^{n}+2 U_{J-3}^{n}\right]$.

(1.7) $U_{0}^{n}+1=U_{0}^{n} 1+\frac{\lambda}{6}\left[-11\left(U_{0}^{n \cdot 1}+U_{0}^{n}{ }^{1}\right)+36 U_{1}^{n}-18 U_{2}^{n}+4 U_{3}^{n}\right]$.

(1) $U_{1}^{\prime \prime}=U_{1}^{\prime \prime 1}+\frac{\lambda}{6}\left[-4 U_{0}^{\prime \prime}-3\left(U_{1}^{n+1}+U_{1}^{n}{ }^{1}\right)+12 U_{2}^{n}-2 U_{3}^{n}\right]$.

The extra conditions are approximations to (1.1) which employ time averaging at $x_{1}, x_{1}$, and $x_{1}$ in (1.6), (1.7) and (1.8). respectively. Each of these conditions has accuracy $O\left(\Delta x^{3}\right)$ in any grid refinement with $\Delta t / \Delta x^{2}$ held constant, and the overall dicuracy of the scheme during such a refinement is $O\left(\Delta x^{4}\right)$.

Here we wish to consider a generalization of the boundary approximations (1.6) (1.8). Gary [3] has pointed out that Eq. (1.6), for example. may be written as $U_{j}^{\prime \prime}:=U_{J}^{\prime \prime}{ }_{1}^{1}+\frac{\lambda}{3}\left(2 U_{J}^{\prime \prime}+3 U_{J}^{n},-6 U_{J}^{n}{ }_{2}+U_{J}^{n}{ }_{3}\right)+\frac{\lambda}{2}\left(U_{J-1}^{n+1}-2 U_{J-1}^{n}+U_{J-1}^{n-1}\right)$. and he has indicated that the final term in this equation may be regarded as a houndary stabilizing term. Gary generalized the concept of adding the boundary stabilizing term by introducing a real parameter $\mu$ to create the modified stabilizing term

$$
\frac{\lambda \mu}{2}\left(U_{J \cdots 1}^{n \cdot 1}-2 U_{J 1}^{n}+U_{J \cdots 1}^{n \cdots 1}\right)
$$

The value $\mu=0$ yields the obvious $O\left(\Delta t^{2}+\Delta x^{3}\right)$ approximation to (1.1) at $x=$ $x, y$ and the value $\mu=1$ yields Oliger's [6] stable approximation. The introduction of $\mu$ to (1.6). (1.7) and (1.8) in this manner produces the modified boundary approximations

(1) 10)

$$
\begin{aligned}
U_{J}^{n+1}= & U_{J}^{n}{ }_{1}^{1}+\frac{\lambda}{3}\left(2 U_{J}^{n}+3 U_{J-1}^{n}-6 U_{J-2}^{n}+U_{J-3}^{n}\right) \\
& +\frac{\lambda \mu}{2}\left(U_{J}^{n+1}-2 U_{J}^{n}{ }_{1}+U_{J}^{n-1}\right) \\
U_{0}^{n+1}= & U_{0}^{n-1}+\frac{\lambda}{3}\left(-11 U_{0}^{n}+18 U_{1}^{n}-9 U_{2}^{n}+2 U_{3}^{n}\right) \\
& \quad \frac{11 \lambda_{\mu}}{2}\left(U_{0}^{n+1}-2 U_{0}^{n}+U_{0}^{n}{ }^{1}\right),
\end{aligned}
$$

$$
\begin{aligned}
U_{1}^{n+1}= & U_{1}^{n-1}+\frac{\lambda}{3}\left(-2 U_{0}^{n}-3 U_{1}^{n}+6 U_{2}^{n}-U_{3}^{n}\right) \\
& -\frac{\lambda \mu}{2}\left(U_{1}^{n+1}-2 U_{1}^{n}+U_{1}^{n-1}\right) .
\end{aligned}
$$

Gary [3] conducted numerical experiments using (1.4), (1.5) and the set of boundary conditions (1.9)-(1.11). He concluded, inter alia, that with $\lambda=0.2$ the difference scheme is stable when $\mu=0.5$ or $\mu=20$, and it is unstable when $\mu=0.4$ or $\mu=25$. His results suggest that the situation here is akin to that which is created by the analogous generalization of the boundary approximation for the second order leap-frog method. Sloan [7] has analyzed the latter problem and he has shown that if 
$\lambda$ satisfies the necessary condition $0<\lambda<1$, there is an interval $\left(\mu_{L}(\lambda), \mu_{u}(\lambda)\right)$, with $0<\mu_{L}(\lambda)<1<\mu_{u}(\lambda)$, such that the second order difference scheme is stable if and only if $\mu \in\left(\mu_{L}, \mu_{u}\right)$. The analysis in [7] also shows that, in terms of accuracy, there is an optimum value of $\mu$ within the interval $\left(\mu_{L}, \mu_{u}\right)$. Here the aim is to produce an analysis of this type for the initial and boundary value difference scheme defined by (1.4), (1.5) and (1.9)-(1.11).

For the fourth order leap-frog method under consideration here the pure Cauchy problem is stable if and only if $0<\lambda<\lambda_{m}$, where $\lambda_{m}=6 /(9+24 \sqrt{6})^{1 / 2} \approx 0.7287$. It is readily shown that the accuracy of the complete difference scheme improves as $\mu$ approaches zero, and if stability holds for $\mu \in\left(\mu_{L}, \mu_{u}\right)$, with $\mu_{L}>0$, then the optimum value of $\mu$ is a value close to $\mu_{L}$. The objective of this paper is to prove that if $\lambda \in\left(0, \lambda_{m}\right)$ there is a value $\mu_{L}(\lambda)>0$ such that the difference scheme defined by (1.4), (1.5) and (1.9)-(1.11) is unstable if $\mu \in\left[0, \mu_{L}(\lambda)\right]$ and stable for $\mu$ in some region with open lower boundary $\mu_{L}(\lambda)$. Values of $\mu_{L}(\lambda)$ will be obtained for a discrete set of $\lambda$ values in $\left(0, \lambda_{m}\right)$. Accuracy diminishes as $\mu$ increases from $\mu_{L}(\lambda)$, so no attempt is made to obtain the upper boundary of the stability interval. For a large part of the interval $0<\lambda<\lambda_{m}$ - but not the complete interval-the value of $\mu_{L}(\lambda)$ is less than unity. When $\mu_{L}(\lambda)<1$ a value of $\mu$ may be selected which will produce more accurate boundary approximations than those analyzed by Oliger [6].

2. Description of the Problem. The local truncation error of each of the boundary approximations (1.9)-(1.11) is readily shown to have the form $O\left(\lambda \mu \Delta t+\Delta t^{2}+\Delta x^{3}\right)$ as $\Delta t \rightarrow 0$ and $\Delta x \rightarrow 0$. This becomes $O\left(\Delta x^{3}\right)$ in any grid refinement in which $\Delta t / \Delta x^{2}$ is held constant. However, for a prescribed $\Delta x$ and an acceptable value of $\lambda \in\left(0, \lambda_{m}\right)$ the local truncation errors will be minimized if $|\mu|$ is minimized subject to stability restrictions. Numerical experiments indicate that for $\lambda \in\left(0, \lambda_{m}\right)$ the scheme is unstable if $\mu \leqslant \mu_{L}(\lambda)$, where $\mu_{L}(\lambda)>0$, and also that the accuracy deteriorates as $\mu$ increases from $\mu_{L}(\lambda)$. The investigation is therefore reduced to a stability analysis and, in particular, to the evaluation of the lower stability limit, $\mu_{L}(\lambda)$.

The stability theory of Gustafsson, Kreiss and Sundström [4] will be used to analyze the stability of the difference problem. The stability of the two-boundary problem is equivalent to the stability of the outflow and inflow quarter-plane problems which are defined, respectively, by removing the right- and left-hand boundaries and extending the domain to $\pm \infty$, as is appropriate. The inflow problem is simplified if we negate the wave speed $c$ and replace the left quarter-plane problem by an equivalent right quarter-plane problem. The inflow problem is therefore defined by

$$
\begin{aligned}
U_{j}^{n+1}= & U_{j}^{n-1}-\frac{\lambda}{6}\left(U_{j-2}^{n}-8 U_{j-1}^{n}+8 U_{j+1}^{n}-U_{j+2}^{n}\right), \quad j \geqslant 2, \\
U_{0}^{n+1}= & g\left(t_{n+1}\right), \\
U_{1}^{n+1}= & U_{1}^{n-1}-\frac{\lambda}{3}\left(-2 U_{0}^{n}-3 U_{1}^{n}+6 U_{2}^{n}-U_{3}^{n}\right) \\
& +\frac{\lambda \mu}{2}\left(U_{1}^{n+1}-2 U_{1}^{n}+U_{1}^{n-1}\right),
\end{aligned}
$$

with $\lambda \in\left(0, \lambda_{m}\right)$. 
To examine the stability of the finite difference schemes we seek a solution of (1.4) or (2.1) in the form $U_{j}^{n}=\alpha z^{n} \kappa^{j}$, where $\alpha, z$ and $\kappa$ are complex numbers. The condition for the existence of a nontrivial solution of (1.4) or (2.1) is that $z$ and $\kappa$ should satisfy the characteristic equations

$$
\kappa^{4}-8 \kappa^{3} \pm \frac{6\left(z^{2}-1\right)}{\lambda z} \kappa^{2}+8 \kappa-1=0 .
$$

This pair of equations has been examined by Oliger [6] in his stability analysis of the boundary conditions corresponding to those considered here under the constraint $\mu=1$. For convenience we quote two results concerning the roots of (2.4) which have been derived by Oliger.

LEMMA 2.1. Let $z$ be a complex number. For $|z| \geqslant 1$, the roots of $(2.4 \mathrm{a}, b)$ split into two groups

$$
M_{1}=\left[\kappa_{1}(z ; \pm \lambda), \kappa_{2}(z ; \pm \lambda)\right] \text { and } M_{2}=\left[\kappa_{3}(z ; \pm \lambda), \kappa_{4}(z ; \pm \lambda)\right]
$$

such that

(i) $\left|\kappa_{1}(z ; \pm \lambda)\right| \leqslant 1,\left|\kappa_{2}(z ; \pm \lambda)\right| \leqslant 1$,

(ii) $\left|\kappa_{3}(z ; \pm \lambda)\right| \geqslant 1, \mid \kappa_{4}(z ; \pm \lambda) \geqslant 1$,

where $\kappa_{i}, i=1,2,3,4$ are continuous functions of $z$. Both of the inequalities (i) and (ii) can be taken strictly if and only if $|z|>1$ or, for $z=e^{i \theta}$ with $|\sin \theta|>\lambda / \lambda_{m}$ and $\lambda_{m}=6 /(9+24 \sqrt{6})^{1 / 2}$. Furthermore, at least one of the inequalities in (i) and in (ii) can always be taken strictly.

LEMMA 2.2. The elements of $M_{1}$ are distinct for $|z| \geqslant 1$, except for the single value $z_{0}$ which is the root of $z^{2} \pm(\lambda / 3)(-9+24 \sqrt{6})^{1 / 2} z-1=0$, such that $\left|z_{0}\right|>1$.

This completes the lemmas.

For the outflow problem $z_{0}$ has the value

$$
-\frac{1}{6}\left[\lambda(-9+24 \sqrt{6})^{1 / 2}+\left\{\lambda^{2}(-9+24 \sqrt{6})+36\right\}^{1 / 2}\right]
$$

and this is negated to give $z_{0}$ for the inflow problem. In either case it is readily shown that the double root within the unit circle in the $\kappa$-plane is given as a root of the cubic

$$
\kappa^{3}-6 \kappa^{2}-(-9+24 \sqrt{6})^{1 / 2} \kappa+2=0
$$

and the value, correct to 4 decimal places, is $\kappa_{0}=0.2374$.

We first consider the stability of the outflow problem. For $|z|>1, z \neq z_{0}$, the general solution of (1.4) which decays as $j$ increases is $U_{j}^{n}=z^{n}\left(c_{1} \kappa_{1}^{j}+c_{2} \kappa_{2}^{j}\right)$, where $\kappa_{1}=\kappa_{1}(z ; \lambda)$ and $\kappa_{2}=\kappa_{2}(z ; \lambda)$ are the roots of $(2.4 \mathrm{a})$ in the set $M_{1}$ and $c_{1}$ and $c_{2}$ are arbitrary complex constants. If this solution is substituted in the boundary approximations (1.10) and (1.11), a homogeneous linear system in $c_{1}$ and $c_{2}$ results and the determinant of the coefficient matrix of this system is

$$
D(z ; \lambda, \mu)=Q_{1}\left(z, \kappa_{1} ; \lambda, \mu\right) Q_{2}\left(z, \kappa_{2} ; \lambda, \mu\right)-Q_{1}\left(z, \kappa_{2} ; \lambda, \mu\right) Q_{2}\left(z, \kappa_{1} ; \lambda, \mu\right),
$$

where

$$
\begin{aligned}
& Q_{1}(z, \kappa ; \lambda, \mu)=\frac{3}{\lambda}\left(z^{2}-1\right)+\frac{11}{2} \mu(z-1)^{2}+z\left(11-18 \kappa+9 \kappa^{2}-2 \kappa^{3}\right), \\
& Q_{2}(z, \kappa ; \lambda, \mu)=\frac{3}{\lambda} \kappa\left(z^{2}-1\right)+\frac{3}{2} \mu \kappa(z-1)^{2}+z\left(2+3 \kappa-6 \kappa^{2}+\kappa^{3}\right)
\end{aligned}
$$


and $\kappa_{1}=\kappa_{1}(z ; \lambda), \kappa_{2}=\kappa_{2}(z ; \lambda) . D(z ; \lambda, \mu)$ is defined for all $|z| \geqslant 1$.

If $z=z_{0}$, then $\kappa_{1}=\kappa_{2}=\kappa_{0}$, and the general solution of (1.4) which decays as $j$ increases is $U_{j}^{n}=z_{0}^{n} \kappa_{0}^{j}\left(c_{1}+j c_{2}\right)$. As before, substitution in (1.10) and (1.11) yields a homogeneous linear system in $c_{1}$ and $c_{2}$ and the determinant of the coefficient matrix is

$$
D(\lambda, \mu)=Q_{1}\left(z_{0}, \kappa_{0} ; \lambda, \mu\right) Q_{4}\left(z_{0}, \kappa_{0} ; \lambda, \mu\right)-Q_{3}\left(z_{0}, \kappa_{0}\right) Q_{2}\left(z_{0}, \kappa_{0} ; \lambda, \mu\right),
$$

where

$$
Q_{3}(z, \kappa)=-6 z \kappa\left(3-3 \kappa+\kappa^{2}\right)
$$

and

$$
Q_{4}(z, \kappa ; \lambda, \mu)=\frac{3}{\lambda} \kappa\left(z^{2}-1\right)+\frac{3}{2} \mu \kappa(z-1)^{2}+3 z \kappa\left(1-4 \kappa+\kappa^{2}\right) .
$$

From the theory of Gustafsson et al. [4] it follows that for $0<\lambda<\lambda_{m}$ and a prescribed real value of $\mu$ the outflow problem is stable if and only if

$$
D(z ; \lambda, \mu)=0
$$

has no solution $z$ in $|z| \geqslant 1$, and, in addition,

$$
D(\lambda, \mu) \neq 0 .
$$

Owing to the complexity of (2.5) this equation has to be analyzed numerically, and the treatment involves the simultaneous solution of $(2.4 \mathrm{a})$ and $(2.5)$ for $\kappa_{1}(z ; \lambda)$, $\kappa_{2}(z ; \lambda)$ and $z$. Values of the triple $\left(\kappa_{1}, \kappa_{2}, z\right)$ are obtained by solving a set of three multivariate polynomial equations. Equation (2.4a) enables us to write two of the polynomial equations as

$$
\begin{aligned}
& \lambda z\left(\kappa_{1}^{4}-8 \kappa_{1}^{3}+8 \kappa_{1}-1\right)+6\left(z^{2}-1\right) \kappa_{1}^{2}=0, \\
& \lambda z\left(\kappa_{2}^{4}-8 \kappa_{2}^{3}+8 \kappa_{2}-1\right)+6\left(z^{2}-1\right) \kappa_{2}^{2}=0 .
\end{aligned}
$$

To complete the system we write Eq. (2.5) as a polynomial in $z$ with coefficients which depend on $\kappa_{1}$ and $\kappa_{2}$. Each coefficient in the polynomial has a factor $\kappa_{2}-\kappa_{1}$ and if this nonzero factor is removed, (2.5) takes the form

$$
a b z^{4}+\mathfrak{A} z^{3}+\mathfrak{B} z^{2}+\mathfrak{S} z+\mathfrak{D}=0,
$$

where $\mathfrak{A}=a A+b B, \mathfrak{B}=a d+b c+C, \mathfrak{S}=c A+d B, \mathfrak{D}=c d$ with $a, c=11 \mu / 2$ $\pm 3 / \lambda ; b, d=3 \mu / 2 \pm 3 / \lambda$ and

$$
\begin{aligned}
A= & \kappa_{1}^{2}+\kappa_{1} \kappa_{2}+\kappa_{2}^{2}-6\left(\kappa_{1}+\kappa_{2}\right)+3(1-\mu), \\
B= & \kappa_{1} \kappa_{2}\left(2 \kappa_{1}+2 \kappa_{2}-9\right)+11(1-\mu), \\
C= & -3 \kappa_{1}^{2} \kappa_{2}^{2}-6(2+\mu) \kappa_{1} \kappa_{2}\left(\kappa_{1}+\kappa_{2}\right)+(15-11 \mu)\left(\kappa_{1}^{2}+\kappa_{1} \kappa_{2}+\kappa_{2}^{2}\right) \\
& +27(3+\mu) \kappa_{1} \kappa_{2}+(66 \mu-84)\left(\kappa_{1}+\kappa_{2}\right)+33(1-\mu)^{2}+36 .
\end{aligned}
$$

If $\left(\kappa_{1}, \kappa_{2}, z\right)$ is a solution triple of (2.7), then this $z$ is an eigenvalue of the difference scheme if $|z| \geqslant 1$ whilst the associated $\kappa_{1}$ and $\kappa_{2}$ are strictly inside the unit circle. The point $z_{0}$ is an eigenvalue if condition (2.6) fails, that is, if $D(\lambda, \mu)=0$. The value $z$ in a solution triple is a generalized eigenvalue if $|z|=1$ and the associated $\kappa_{1}$ and $\kappa_{2}$ are in the set $M_{1}$ of Lemma 2.1, with $\left|\kappa_{1}\right|=1$ or $\left|\kappa_{2}\right|=1$. In this case, if $z$ is slightly perturbed so that $|z|>1$, the associated $\kappa_{1}$ and $\kappa_{2}$ given by $(2.7 \mathrm{a}, \mathrm{b})$ must be strictly inside the unit circle. The outflow problem is stable if there is no eigenvalue or generalized eigenvalue in $|z| \geqslant 1$. 
The formulation of the inflow stability problem is effected in an analogous manner. In this case, for $0<\lambda<\lambda_{m}$ and a prescribed real value of $\mu$ the inflow problem is stable if and only if

$$
E(z ; \lambda, \mu)=0
$$

has no solution $z$ in $|z| \geqslant 1$, and, in addition,

$$
E(\lambda, \mu) \neq 0 \text {, }
$$

where

$$
\begin{aligned}
E(z ; \lambda, \mu) & =Q_{2}\left(z, \kappa_{2} ;-\lambda, \mu\right)-Q_{2}\left(z, \kappa_{1} ;-\lambda, \mu\right) . \\
E(\lambda, \mu) & =Q_{4}\left(z_{0}, \kappa_{0} ;-\lambda, \mu\right) .
\end{aligned}
$$

Here $\kappa_{1}=\kappa_{1}(z ;-\lambda)$ and $\kappa_{2}=\kappa_{2}(z ;-\lambda)$ are the roots of $(2.4 \mathrm{~b})$ in the set $M_{1}$, and $z_{0}$ and $\kappa_{0}$ are the real positive numbers described by Lemma 2.2. Equation (2.8) has to be solved numerically and the analysis follows that described for (2.5). $\kappa_{1}, \kappa_{2}$ and $z$ are determined by the multivariate system of polynomial equations

$$
\begin{aligned}
& -\lambda z\left(\kappa_{1}^{4}-8 \kappa_{1}^{3}+8 \kappa_{1}-1\right)+6\left(z^{2}-1\right) \kappa_{1}^{2}=0, \\
& -\lambda z\left(\kappa_{2}^{4}-8 \kappa_{2}^{3}+8 \kappa_{2}-1\right)+6\left(z^{2}-1\right) \kappa_{2}^{2}=0, \\
& d z^{2}+\left[\kappa_{1}^{2}+\kappa_{1} \kappa_{2}+\kappa_{2}^{2}-6\left(\kappa_{1}+\kappa_{2}\right)+3(1-\mu)\right] z+b=0,
\end{aligned}
$$

where $d$ and $b$ are defined in (2.7c). The inflow problem is stable if (2.9) holds and if the numerical solution of (2.10) shows that the difference scheme has no eigenvalues or generalized eigenvalues in $|z| \geqslant 1$.

To facilitate the test for generalized eigenvalues which could arise in the numerical solution of (2.7) and (2.10) a few observations should be made concerning the roots of (2.4) which belong to the set $M_{1}$. Note initially that $\kappa(z ; \lambda)$ is a root of $(2.4 \mathrm{a})$ if and only if $\kappa(-z ; \lambda)$ is a root of $(2.4 b)$, so observations need only be made on the outflow roots. Furthermore, $\kappa(z ; \lambda)=\overline{\kappa(\bar{z} ; \lambda)}$, and it should therefore suffice to comment on the roots of $(2.4 \mathrm{a})$ for $z$ in the region

$$
R=\{z: z \in \mathbf{C},|z| \geqslant 1, \operatorname{Im}(z) \geqslant 0\}
$$

of the complex plane C. It follows from Lemma 2.1 that for $z \in R$ generalized eigenvalues can only occur for $z=e^{i \theta}$, with $0 \leqslant \theta \leqslant \theta_{0}$ or $\pi-\theta_{0} \leqslant \theta \leqslant \pi$, where $\theta_{0}=\sin ^{-1}\left(\lambda / \lambda_{m}\right)$. If $\theta$ is restricted in this way, then $\kappa_{1}\left(e^{i \theta} ; \lambda\right)=e^{i \phi}$ and $\kappa_{2}\left(e^{i \theta} ; \lambda\right)$ is strictly inside the unit circle. The symbol $\kappa_{1}$ is used to denote the root of modulus unity for $z$ on either of the two circular arcs. The following lemma contains some observations on the root $\kappa_{1}\left(e^{i \theta} ; \lambda\right)=e^{i \phi}$ for $\theta \in\left[0, \theta_{0}\right]$ or $\theta \in\left[\pi-\theta_{0}, \pi\right]$.

LEMMA 2.3. (i) $\kappa_{1}(1 ; \lambda)=-1$;

(ii) $\kappa_{1}(-1 ; \lambda)=+1$;

(iii) $\kappa_{3}\left(e^{i(\pi-\theta)} ; \lambda\right)=\kappa_{1}\left(e^{i \theta} ; \lambda\right)$ for $\theta \in\left[0, \theta_{0}\right]$ or $\theta \in\left[\pi-\theta_{0}, \pi\right]$. Here $\kappa_{3}$ is a root from the outer set $M_{2}$;

(iv) $\theta \in\left[0, \theta_{0}\right] \Rightarrow \phi \in\left[\phi_{0}, \pi\right]$ and $\theta \in\left[\pi-\theta_{0}, \pi\right] \Rightarrow \phi \in\left[0, \phi_{0}\right]$, where $\phi_{0}=$ $\cos ^{-1}(1-\sqrt{3 / 2})$. In each of these regions the derivative of $\phi$ with respect to $\theta$ is strictly negative.

Proof. The existence of the roots of (2.4a) at $\kappa= \pm 1$ when $z= \pm 1$ is obvious. The identification of the inner root $\kappa_{1}$ is achieved by considering a perturbation of $z$ 
from \pm 1 along the real axis. Result (iii) follows from the invariance of the equation

$$
\kappa^{4}-8 \kappa^{3}+\frac{12 i \sin \theta}{\lambda} \kappa^{2}+8 \kappa-1=0
$$

when $\theta$ is replaced by $\pi-\theta$. Result (iv) is obtained in a straightforward manner from the analysis of roots of this quartic which have the form $\kappa=e^{i \phi}$, for $|(6 \sin \theta) / \lambda| \leqslant(9+24 \sqrt{6})^{1 / 2}$. Note that $\kappa_{1}$ and $\kappa_{3}$ coincide when $\theta=\theta_{0}$ or $\theta=\pi-$ $\theta_{0}$.

If a triple $\left(\kappa_{1}, \kappa_{2}, z\right)$ arises in the numerical solution of (2.7) or (2.10) with $|z|=1$ and $\left|\kappa_{i}\right|=1$ for $i=1$ or 2 , then Lemma 2.3 will enable us to decide if this root corresponds to a generalized eigenvalue.

3. Stability Analysis. The conditions (2.6) and (2.9) were checked numerically by evaluating $D(\lambda, \mu)$ and $E(\lambda, \mu)$ on the grid of values $\lambda=0.05(0.05) 0.70, \mu=$ $0(0.2) 1.0$ within $\left(0, \lambda_{m}\right) \times[0,1]$. Values of $D(\lambda, \mu)$ and $E(\lambda, \mu)$ for $\lambda=0.05$ are shown in Table 3.1 .

\section{TABLE 3.1}

$D(0.05, \mu)$ and $E(0.05, \mu)$ in Eqs. (2.6) and (2.9), for $\mu=0(0.2) 1.0$.

\begin{tabular}{|c|c|l|l|l|l|l|}
\hline$\mu$ & 0 & \multicolumn{1}{c|}{0.2} & \multicolumn{1}{c|}{0.4} & \multicolumn{1}{c|}{0.6} & \multicolumn{1}{c|}{0.8} & \multicolumn{1}{c|}{1.0} \\
\hline$D(\lambda, \mu)$ & 2.40 & 10.6 & 21.7 & 35.5 & 52.2 & 71.7 \\
$E(\lambda, \mu)$ & -1.6960 & -1.6957 & -1.6955 & -1.6952 & -1.6949 & -1.6947 \\
\hline
\end{tabular}

As $\lambda$ increases $D(\lambda, \mu)$ increases and $E(\lambda, \mu)$ decreases for each value of $\mu$, and we therefore conclude that the inequalities (2.6) and (2.9) are satisfied.

The systems of polynomial equations (2.7) and (2.10) were solved numerically using a homotopy continuation algorithm. Each of these equations may be regarded as a system of six real polynomial equations in six real variables, and we therefore consider a method for the numerical solution of an equation of the form

$$
F(X)=0
$$

where $F: \mathbf{R}^{\nu} \rightarrow \mathbf{R}^{\nu}$ is a nonlinear mapping, $\mathbf{R}$ is the set of real numbers and $\nu$ is a positive integer. In a continuation method $F(X)$ is partitioned in the form $F(X)=$ $\Phi(X)+\Psi(X)$, and the problem is embedded in the family of problems

$$
H(Y) \equiv H(X, T) \equiv \Phi(X)+T \Psi(X)=0,
$$

where $T \in \mathbf{R}$, so that $H: \mathbf{R}^{\nu+1} \rightarrow \mathbf{R}^{\nu}$. The vectors $X$ which satisfy (3.1) are solutions of $H(X, 1)=0$, and it is assumed that $F$ has been partitioned such that all solutions of $H(X, 0)=0$ are readily obtained. Various continuation methods have been proposed and the reader is referred to the text by Wacker [8] and the review paper by Allgower and Georg [1].

The problem under consideration here was solved using an adaptation of the algorithms described by Garcia and $\mathrm{Li}$ [2] and $\mathrm{Li}$ and Yorke [5]. Suppose $\eta: \mathbf{R} \rightarrow \mathbf{R}^{\nu+1}$ is a smooth curve such that

(i) $\|\dot{\eta}(s)\|=1$ for $s \in \mathbf{R}$ ( $s$ is arc length), where $\dot{\eta}(\cdot)=d \eta(\cdot) / d s$ and $\|\cdot\|$ is the Euclidean norm;

(ii) $H(\eta(s))=0 \forall s \in \mathbf{R}$; 
(iii) $H^{\prime}(\eta(s))$ has rank $\nu$ for $s \in \mathbf{R}$, where $H^{\prime}$ is the Fréchet derivative of $H$ : all points on the curve are regular.

Here the final component of the vector $\eta(s)$ is the real parameter $T(s)$, and the aim is to follow the homotopy path from the known point at which $T=0$ to the required point at which $T=1$. The path in $\mathbf{R}^{\nu+1}$ is obtained using a predictor-corrector method to solve the initial value problem

$$
\begin{gathered}
H^{\prime}(\eta(s)) \dot{\eta}(s)=0, \\
\|\dot{\eta}(s)\|=1,
\end{gathered}
$$

from an initial value $\eta(0)=Y_{0}$, where $Y_{0}=\left(X_{0}, 0\right)$ and $X_{0}$ is one of the known solutions of $\Phi(X)=0$. A Runge-Kutta predictor finds an approximation by moving a distance $\Delta s$ along a suitable unit vector $B$ from a known point on the homotopy path, and a Newton corrector is then used to solve the integrated system (3.2). The iteration is constructed so that successive iterates lie on a hyperplane perpendicular to the unit vector $B$.

In the adaptation of the above algorithm which was used to solve (2.7) and (2.10) the operator $H^{\prime}$ is the $3 \times 4$ complex matrix of partial derivatives of $H$ with respect to $\kappa_{1}, \kappa_{2}, z$ and $T$. (3.3a) is solved to give $\dot{\kappa}_{1}(s), \dot{\kappa}_{2}(s), \dot{z}(s)$ and $\dot{T}(s)$ as $3 \times 3$ complex determinants, and each component of this solution is then multiplied by the complex conjugate of the solution for $\dot{T}(s)$ to give a quadruple $\left(\dot{\kappa}_{1}(s), \dot{\kappa}_{2}(s), \dot{z}(s), \dot{T}(s)\right)$ in which $\dot{T}(s)$ is real and strictly positive $\forall s \in \mathbf{R}$. The algorithm is modified to yield $\eta: \mathbf{R} \rightarrow \mathbf{C}^{3} \times \mathbf{R}$, where the components of $\eta(s)$ are $\kappa_{1}(s), \kappa_{2}(s), z(s)$ and $T(s)$.

The partitioning adopted for the solution of the outflow stability equations (2.7) was

$$
\begin{aligned}
\kappa_{1}^{5}-1+T\left[-\kappa_{1}^{5}+\lambda z\left(\kappa_{1}^{4}-8 \kappa_{1}^{3}+8 \kappa_{1}-1\right)+6\left(z^{2}-1\right) \kappa_{1}^{2}+1\right] & =0 \\
\kappa_{2}^{5}-1+T\left[-\kappa_{2}^{5}+\lambda z\left(\kappa_{2}^{4}-8 \kappa_{2}^{3}+8 \kappa_{2}-1\right)+6\left(z^{2}-1\right) \kappa_{2}^{2}+1\right] & =0 \\
z^{6}+1+T\left[-z^{6}+z^{4}+\left(\mathfrak{A} z^{3}+\mathfrak{B} z^{2}+(5 z+\mathfrak{D}) /(a b)-1\right]\right. & =0
\end{aligned}
$$

When $T=0$ the degrees of the component equations in (3.4) are 5,5 and 6 and these match the degrees of the component equations in the multivariate system (2.7). The solutions of (3.4) at $T=0$ give rise to 150 initial values $\eta(0)=\left(\kappa_{1}(0), \kappa_{2}(0), z(0), 0\right)$ for the system (3.3). The use of symmetry in $\kappa_{1}$ and $\kappa_{2}$ and the elimination of complex conjugates enables us to reduce this to a set of 30 initial values. In (3.4) we selected a value of $\lambda$ in $\left(0, \lambda_{m}\right)$, set $\mu=0.1$ and applied the continuation algorithm using each of the 30 initial vectors. This produced 8 solution triples for (2.7), and in this solution set the only unstable triple was that obtained from the branch with initial value $\left(e^{2 \pi i / 5}, e^{-4 \pi i / 5}, e^{5 \pi i / 6}, 0\right)$. Solutions were then obtained using this initial value with $\mu=0.1,0.2, \ldots$, and the solution procedure was terminated when $|z|$ in the solution triple fell below the value unity. Table 3.2 shows the unstable triple for $\lambda=0.5$ and $\mu=0.1(0.1) 0.6$. 
TABLE 3.2

Unstable triple for outflow problem with $\lambda=0.5$.

\begin{tabular}{|c|l|l||l|l||l|l||c|}
\hline$\mu$ & $\operatorname{Re}\left(\kappa_{1}\right)$ & $\operatorname{Im}\left(\kappa_{1}\right)$ & $\operatorname{Re}\left(\kappa_{2}\right)$ & $\operatorname{Im}\left(\kappa_{2}\right)$ & $\operatorname{Re}(z)$ & $\operatorname{Im}(z)$ & $|z|$ \\
\hline 0.1 & 0.4146 & 0.6277 & 0.1232 & -0.02476 & -1.0094 & 0.5592 & 1.1539 \\
0.2 & 0.2290 & 0.6999 & 0.1189 & -0.02751 & -0.9160 & 0.6858 & 1.1443 \\
0.3 & 0.09252 & 0.7216 & 0.1162 & -0.02825 & -0.8332 & 0.7430 & 1.1164 \\
0.4 & -0.01937 & 0.7179 & 0.1144 & -0.02824 & -0.7626 & 0.7619 & 1.0780 \\
0.5 & -0.1158 & 0.6963 & 0.1131 & -0.02780 & -0.7029 & 0.7539 & 1.0307 \\
0.6 & -0.2009 & 0.6584 & 0.1120 & -0.02700 & -0.6523 & 0.7230 & 0.9738 \\
\hline
\end{tabular}

A bisection technique was used to locate the value $\mu=\mu_{L}$ at which $|z|=1$, and stability was checked for $\mu$ slightly larger than $\mu_{L}$ by applying the continuation algorithm on all 30 initial quadruples for the system (3.3). Table 3.2 indicates that as $\mu$ passes through $\mu_{L}$ the point $z$ crosses the unit circle at a point $e^{i \theta}$ with $\sin \theta>\sin \theta_{0}$. Table 3.3 shows $\mu_{L}$ correct to two significant figures for a set of $\lambda$ values in $\left(0, \lambda_{m}\right)$.

TABLE 3.3

Stability limit $\mu_{L}$ for the outflow problem.

\begin{tabular}{|c|l|l|l|l|l|l|l|l|l|}
\hline$\lambda$ & 0.1 & 0.2 & 0.3 & 0.4 & 0.5 & 0.6 & 0.65 & 0.675 & 0.68 \\
$\mu_{L}$ & 0.47 & 0.48 & 0.49 & 0.52 & 0.56 & 0.64 & 0.73 & 0.88 & UNS \\
\hline
\end{tabular}

The entry UNS under $\lambda=0.68$ indicates that $\mu_{L}>1$ and that the outflow problem is therefore unstable when $\mu=1$. This contradicts Oliger's [6] conclusion that the difference problem which he examined is stable for all $\lambda \in\left(0, \lambda_{m}\right)$.

An analysis of system (2.10) by the continuation method indicated that the inflow problem is stable for all $\mu \in[0,1]$ and $\lambda \in\left(0, \lambda_{m}\right)$. We therefore conclude that Table 3.3 gives the lower limit on $\mu$ for stability of the initial and boundary value difference problem defined by (1.4), (1.5) and (1.9)-(1.11). The results in Table 3.3 agree with the computational results of Gary [3]. With $\lambda=0.4$ Gary found the problem to be stable at $\mu=0.6$ and unstable at $\mu=0.5$ and with $\lambda=0.2$ he found stability at $\mu=0.5$ and instability at $\mu=0.4$.

4. Numerical Results. To support the analysis in the preceding sections the problem defined by Eqs. (1.1)-(1.3) was solved numerically using the difference scheme (1.4), (1.5) and (1.9)-(1.11), and the results were compared with the theory. The boundary functions $g$ and $q$ were initially defined by $g(t)=\sin 4 \pi(1+t)$, $q(x)=\sin 4 \pi x$, and the wave speed $c$ was set to unity. In Table 4.1, where results are given for $J=20$, the solution growth is conveniently illustrated by means of the norm

$$
\left\|U\left(t_{n}\right)\right\|_{2}^{2}=\Delta x \sum_{j=0}^{J} \sigma_{j}\left[U_{j}^{n}\right]^{2}
$$


where $\sigma_{j}=0.5$ for $j=0, J$ and $\sigma_{j}=1$ otherwise. This is a discrete approximation to the quantity

$$
\int_{0}^{1} u^{2}(x, t) d x
$$

which has the constant value 0.5 for the solution $u(x, t)=\sin 4 \pi(x+t)$. Results are given for values of $n$ up to 2000, and if a solution is growing the entries in Table 4.1 are terminated at a value of $n$ where the instability has become obvious. An entry in brackets such as $(+3)$ represents a decimal exponent.

\section{TABLE 4.1 $(a)$}

$\left\|U\left(t_{n}\right)\right\|_{2}^{2} \times 10^{3}$ for exact solution $u(x, t)=\sin 4 \pi(x+t)$ with $J=20, \lambda=0.4$.

\begin{tabular}{|c|c|l|l|l|l|l|l|l|l|l|}
\hline$\mu$ & $n: 200$ & 400 & 600 & 800 & 1000 & 1200 & 1400 & 1600 & 1800 & $2(000$ \\
\hline 0.48 & 484 & 504 & $148(+2)$ & $545(+4)$ & $201(+7)$ & & & & & \\
0.50 & 484 & 482 & 485 & 496 & 624 & $262(+1)$ & $253(+2)$ & $207(+3)$ & $205(+4)$ & $421(+5)$ \\
0.52 & 484 & 482 & 484 & 485 & 484 & 489 & 488 & 482 & 483 & 466 \\
0.54 & 483 & 482 & 483 & 484 & 484 & 485 & 485 & 483 & 483 & 481 \\
\hline
\end{tabular}

\section{TABLE 4.1(b)}

$\left\|U\left(t_{n}\right)\right\|_{2}^{2} \times 10^{3}$ for exact solution $u(x, t)=\sin 4 \pi(x+t)$ with $J=20, \lambda=0.6$.

\begin{tabular}{|c|c|l|c|l|c|c|c|c|c|c|}
\hline$\mu$ & $n: 200$ & \multicolumn{1}{c|}{400} & 600 & \multicolumn{1}{c|}{$8(0)$} & $10(0)$ & 1200 & 1400 & 1600 & $18(0)$ & $2(0)(0)$ \\
\hline 0.60 & 592 & $809(+4)$ & $213(+9)$ & & & & & & & \\
0.62 & 463 & 602 & $195(+2)$ & $251(+4)$ & $323(+6)$ & $418(+8)$ & & & & \\
0.64 & 460 & 460 & 460 & 460 & 460 & 460 & 460 & 460 & 460 & 460 \\
0.66 & 458 & 459 & 459 & 459 & 459 & 459 & 459 & 459 & 459 & 459 \\
\hline
\end{tabular}

TABLE 4.1(c)

$\left\|U\left(t_{n}\right)\right\|_{2}^{2}$ for exact solution $u(x, t)=\sin 4 \pi(x+t)$ with $J=20, \lambda=0.7, \mu=1$.

\begin{tabular}{|c|c|c|}
\hline$n: 200$ & 400 & 600 \\
$146(+12)$ & $416(+30)$ & $549(+48)$ \\
\hline
\end{tabular}

Tables 4.1(a,b) show clearly the change from instability to stability as $\mu$ increases through the value $\mu_{L}$ and Table 4.1(c) shows the aforementioned instability at $\lambda=0.7 \in\left(0, \lambda_{m}\right)$ when $\mu=1$. Table 4.1(b) indicates that the deviation of $\left\|U\left(t_{n}\right)\right\|_{2}^{2}$ from 0.5 increases as $\mu$ increases from $\mu_{L}$. To show the variation of accuracy with $\mu$ in terms of the maximum norm

$$
e\left(t_{n}\right)=\max _{j}\left|U_{j}^{n}-u\left(x_{j}, t_{n}\right)\right|
$$

we have given results in Table 4.2 for the problem with exact solution $u(x, t)=x+$ $t+\exp (-x-t)$. This choice should prevent misinterpretation due to error oscillation. 
TABLE 4.2

$e\left(t_{n}\right)$ for exact solution $u(x, t)=x+t+\exp (-x-t)$ with $J=20, \lambda=0.25$.

\begin{tabular}{|c|c|c|c|c|}
\hline$\mu$ & $n: 80$ & 160 & 240 & 320 \\
\hline 0.5 & $283(-7)$ & $980(-8)$ & $480(-8)$ & $274(-8)$ \\
0.6 & $318(-7)$ & $121(-7)$ & $512(-8)$ & $255(-8)$ \\
0.7 & $359(-7)$ & $141(-7)$ & $555(-8)$ & $285(-8)$ \\
0.8 & $399(-7)$ & $161(-7)$ & $596(-8)$ & $323(-8)$ \\
0.9 & $440(-7)$ & $182(-7)$ & $637(-8)$ & $387(-8)$ \\
1.0 & $482(-7)$ & $203(-7)$ & $693(-8)$ & $471(-8)$ \\
\hline
\end{tabular}

Table 4.2 shows clearly that $\mu$ should be chosen close to the stability limit $\mu_{L}$.

The study has shown how a simple modification may be used to improve the time-averaged boundary conditions which were proposed by Oliger [6]. It is hoped that the work described here will prove useful in the construction of boundary conditions for more general hyperbolic systems. Gary [3] has pointed out that the addition of stabilizing terms to boundary approximations is readily accomplished in the case of nonlinear situations. Oliger [6] has used his time-averaging technique for linear hyperbolic systems in one and two space dimensions.

Department of Mathematics

University of Strathclyde

Glasgow G1 1XH, Scotland

1. E. Allgower \& K. Georg, "Simplicial and continuation methods for approximating fixed points," SIAM Rev., v. 22, 1980, pp. 28-85.

2. C. B. Garcia \& T. Y. Li, On a Path Following Method for Systems of Equations, Report 1983, MRC, University of Wisconsin, Madison, 1979.

3. J. GARY. "On boundary conditions for hyperbolic difference schemes," J. Comput. Phys., v. 26, 1978, pp. 339-351.

4. B. Gustafsson, H.-O. KReISS \& A. SUNDSTRÖM, "Stability theory of difference approximations for mixed initial boundary value problems. II," Math. Comp., v. 26, 1972, pp. 649-686.

5. T. Y. Li \& J. A. YoRKe, A Simple Reliable Numerical Algorithm for Following Homotopy Paths, Report 1984, MRC, University of Wisconsin, Madison, 1979.

6. J. OLIGER, "Fourth order difference methods for the initial boundary-value problem for hyperbolic equations," Math. Comp., v. 28, 1974, pp. 15-25.

7. D. M. SLOAN, "Stability and accuracy of a class of numerical boundary conditions for the advection equation," IMA J. Numer. Anal., v. 1, 1981, pp. 285-301.

8. H. WACKer, Continuation Methods, Academic Press, New York, 1978. 